\title{
WCC 2016-102; OUTCOME OF COMPLETE HEART BLOCK IN ACUTE MYOCARDIAL INFARCTION
}

\author{
Ramakrishna Janapati
}

\section{Introduction:}

Heart blocks may occur as a complication of acute myocardial infarction (AMI) and are accompanied by increased in-hospital mortality. The objective of this present study was to observe the improvement of complete heart block with or without revascularization using thrombolytic or primary or delayed PCI in cases acute coronary syndrome during in hospital extended follow up.

\section{Methods:}

Thirty consecutive acute myocardial infarction patients who were complicated by complete heart block were included in the present study, between January 2014 to July 2015. All CHB patients whether thrombolysed or not irrespective of the duration of the chest pain were included in the study. Temporary Pacemakers were implanted in the majority of patients.

\section{Results:}

Total number of study population was 30. Mean age of the subject was 61 years and 19 patients were male and 11 female patients were included. Of them.26 patients presented with chest pain, 4 patients with SOB and 4 patients presented with syncope. The incidence of risk factors was: type 2 DM in 16, HTN in 24 and smoking in 12. Out of them 12 patients presented with cardiogenic shock.All of them had CHB at presentation with junctional escape rate of $45 \pm 3$ beats/min. Inferior wall MI was more frequent( $20 / 30)$ than AWMI (10/30).AWMI is associated with moderate to severe LV dysfunction and out of 10 patients 7 patients were in cardiogenic shock and 5 of them required IABP support .Out of 30 patients 24 patients required Temporary pacemakers and 6 patients had transient CHB which reverted to normal sinus rhythm before reaching to our hospital all the patients were IWMI patients. Mean duration of TPI requirement was 6.4 days. Only 2 patients required Permanent pacemaker implantation .Death occurred in 60\% AWMI patients and 10\% IWMI patients. RCA was the culprit vessel in 16 patients LCX in 5 patients and LAD was the culprit vessel in 9 patients.

\section{Conclusion:}

$\mathrm{CHB}$ was more commonly seen in IWMI. The recovery to sinus rhythm was more frequently observed in revascularization group irrespective of revascularization strategy .AWMI with CHB is more lethal. Early detection and prompt revascularization reduces the morbid and mortality. 